\title{
Fuzzy Semantic Web Architecture for Activity Detection in Wireless Multimedia Sensor Network Applications
}

\author{
Ali Nail Ozdin ${ }^{a}$ and Adnan Yazici ${ }^{b}$ and Murat Koyuncu ${ }^{c}$
}

aDept. of Computer Engineering, Middle East Technical University, Ankara, Turkey, alinailozdin@gmail.com

bept. of Computer Science, Nazarbayev University, Astana, Kazakhstan, adnan.yazici@nu.edu.kz

cDept. of Information Systems Engineering, Atilim University, Ankara, Turkey, mkoyuncu@atilim.edu.tr

\begin{abstract}
This study aims to increase the reliability of activity detection in Wireless Multimedia Sensor Networks (WMSNs) by using Semantic Web technologies extended with fuzzy logic. The proposed approach consists of three layers: the sensor layer, the data layer, and the Semantic Web layer. The sensor layer comprises a WMSN comprising sensor nodes with multimedia and scalar sensors. The data layer retrieves and stores data from the sink of WMSN. At the top of the architecture, there is a semantic web layer that includes a semantic web application server, a fuzzy reasoning engine, and a semantic knowledge base. When a new entity is detected at the sensor layer, the associated data produced by the sensors and the sink are collected in the data layer and transmitted to the semantic web application server where the data is converted into subjects, predicates, and objects, according to the ontology conceived and recorded in RDF format. Then, the fuzzy reasoning engine is automatically activated and fuzzy rules are executed to determine if there is an activity in the monitored area. Our implementation confirms that extended semantic Web technologies with fuzzy logic can have a significant impact on the detection of activities in WMSNs.
\end{abstract}

Keywords: Fuzzy logic, Semantic Web, Activity detection, Wireless multimedia sensor networks.

\section{Introduction}

The rapid development of microelectronics has led to an increase in the growth of Wireless Multimedia Sensor Networks (WMSNs) for surveillance applications [1]. In this area, activity detection has become an important research topic in recent years [9]. Activities can be obtained from environmental measurements made by scalar sensors such as PIR, acoustic and vibratory sensors, as well as multimedia sensors such as a camera and a microphone. The difficulty of determining an activity depends on the type of activities to be determined. Identifying an activity involves distinguishing between similar activities and deciding on the type of activity.

According to Berners-Lee et al. [5], the Semantic Web is a continuation of Web 2.0 and allows computers and people to collaborate with semantically well-defined information. It aims to explain the meaning of the data. In addition, the Semantic Web makes it possible to combine different sources of data and to obtain new data by reasoning from these sources [4]. It is therefore possible to use this technology for activity detection using a rule-based system [22]. On the other hand, it is very convenient to use Fuzzy Set Theory to increase the reliability of a rule-based system. It is more appropriate to use reasoning and uncertainty based on intuitive reasoning [28]. The reasoning may not consist of two outcomes [25]. On the basis of the knowledge acquired by domain experts, it is possible to decide whether an event occurs with a possibility of zero, one or an intermediate value.

This study presented in this paper proposes an approach for integrating WMSNs and Semantic Web technologies extended with fuzzy logic in order to: 1) use fuzzy reasoning for more reliable detection of activities; 2) leverage the Semantic Web for sharing WMSN data in a well-defined format so that end-users and other systems can make use of this data. Our proposed approach consists of three layers, namely the sensor, data and semantic Web layers. The sensor layer covers the environment in which the wireless multimedia sensor nodes, the gateways, and the sink are deployed. In the second layer, the data detected by the sensors and pre-processed by the sensor nodes and/or the sink, which are ready to be used for activity detection or to be shared, are stored. At the top of the architecture, there is a semantic web layer that includes a semantic web application server, a fuzzy reasoning engine, and a semantic knowledge base. When a new data item reaches the Semantic Web Application Server, it is converted to semantic Web 
tuples (subjects, predicates, and objects) and stored in Resource Description Framework (RDF) format. Then, the fuzzy reasoning engine is activated and the associated rules are triggered for activity detection. As part of putting this approach into practice, a surveillance case study called Border Safety Informer (BSI) is implemented. This application simulates a WMSN deployed around a border post and attempts to detect threats (attack, manifestation, or ordinary mobility), based on various levels of concern.

This study introduces a number of contributions. The first contribution of this study is the translation of processed sensor data obtained from a WMSN into semantic web data in order to make them available for other applications or end users. The second contribution is the integration of fuzzy logic into the reasoning system included in the Semantic Web. There are no studies in the literature using these two main functionalities together [4, 7, 17, 29].

The rest of the paper is organized as follows: The following section provides an overview of the corresponding work. In Section 3, we introduce the architecture for activity detection in the WMSN applications. For proof of concept, a case study based on a simulated synthetic data set is presented in Section 4. Finally, conclusions are drawn in Section 5.

\section{Related Work}

Over the last decade, Semantic Web technologies have been integrated into wireless sensor networks (WSN) for various purposes [27], such as activity detection and data management [24], raw sensor data processing [3], analysis of sensor networks and their data [21], optimization of energy consumption of sensor networks [6], or estimation of fire wheather indices [14].

Activity detection can be done using semantic web technology as well as other technologies. For example, the study presented in [17] performs activity detection by applying dynamic K-means clustering. For a smart home system, they place PIR motion sensors at various locations in a house and object sensors on certain objects, such as a phone. Seven activities are defined: sweeping, cooking, washing, cleaning, eating, sleeping and talking. Using the K-means classification, the first four activities are attributed to the passive learning system and the last three activities should be learned by the system. In addition, the study in [8] deals with the detection of voice activity and uses the SVM and GMM classifiers to label and classify acoustic events (applause, cup sound, etc.). After the classification, the voice activity detection is performed by separating the acoustic event sounds from the speech. The study presented in [23] focuses on monitoring the activity in smart home systems that has emerged as a result of widespread use of healthcare monitoring applications. Supervised activity detection is used in the study. Another study
[13] focuses on the recognition of unsupervised activities because of the high cost of manual labeling. In order to achieve unsupervised activity recognition with the framework called NECTAR, a hybrid approach consisting of ontology and segmentation was introduced. The segments, via the comments of the environment to avoid errors due to heterogeneous environmental conditions, consist of sensor events that cannot be defined by the ontology.

A study performed by Zafeiropoulos et al. investigates data management on sensor networks using semantic web technologies [29]. The problem pointed out by them is that, due to the lack of ontological definitions, end-users cannot reach the required information by using raw data produced by the sensor networks. As a solution, they propose a 3-layered architecture: a data layer that collects and groups sensor data, a processing layer that processes the grouped data, and finally, a semantic layer that is responsible for context annotation and reasoning. The study covers only the classical semantic web rule-based reasoning technique without Fuzzy Set Theory while detecting activities. Similarly, Moraru et al. propose a framework that automatically converts sensor raw data to a comprehensive semantic sensor data [24]. They aim to automatically annotate sensor data, publish sensor data using well-known vocabulary and support event detection applications by applying reasoning techniques. Gómez-Romero et al. propose an ontology-based extension for traditional tracking system to minimize tracking errors [15]. Ontologies are used to build abstract descriptions of the scene, in terms of symbolic entities. These descriptions are the input of the reasoning procedures, which detect or predict tracking errors that are inconsistent with the current situation, and alert the tracking algorithm. Later, they extended the study and converted it to a fusion-based threat assessment system for harbor surveillance [16]. The system architecture is organized into two levels: the first uses an ontological model to formally represent input data and to classify the harbor objects and basic situations by deductive reasoning according to the harbor regulations. The second level applies Belief-based argumentation to assess the threat posed by suspicious vessels.

There are also some studies using the Semantic Web not for activity detection but for other purposes [4,7]. These studies use different types of wireless sensor networks and semantic Web services. For example, the study specified in [4] introduces two approaches that allow different types of sensor networks to communicate. The first method is that each node can have its own semantic Web service. It is not necessary to have a generic Web service to publish the data of the node. The second method is to have a base station and deploy the semantic Web service on the base station to communicate with different sensor networks. In this case, requests are issued by end-users using third-party software, the corresponding 
responses are retrieved from the base station and forwarded to the end-users [4]. Similarly, [18] also aims to reuse data produced by different sensor networks that perform measurements such as sound, location and temperature between different applications. Kapitanova et al. put forward a study that makes event detection in wireless sensor networks with fuzzy set theory [19]. Their study aims to tolerate data from untrusted and low-precision sensor using the fuzzy set theory.

\section{Architecture}

The purpose of this study is to design a reliable reasoning system for use in activity monitoring applications and to make the acquired data available to other users or applications. To achieve this goal, semantic Web technologies extended by fuzzy-set theory are integrated into a WMSN that enables environmental monitoring effectively. Figure 1 shows the proposed system architecture composed of three main layers. Sensors, living things and their environment form the sensor layer. The raw data detected by these sensors are processed at the sensor nodes and/or the sink to determine if there is a target object in the monitoring area. This data is then stored in the data layer. Finally, this processed data is transferred to the semantic Web layer. The Semantic Web Layer manages the entire Semantic Web and its fuzzy reasoning process for activity detection. It is extended to the theory of fuzzy sets to obtain a reasoning mechanism close to human reasoning.

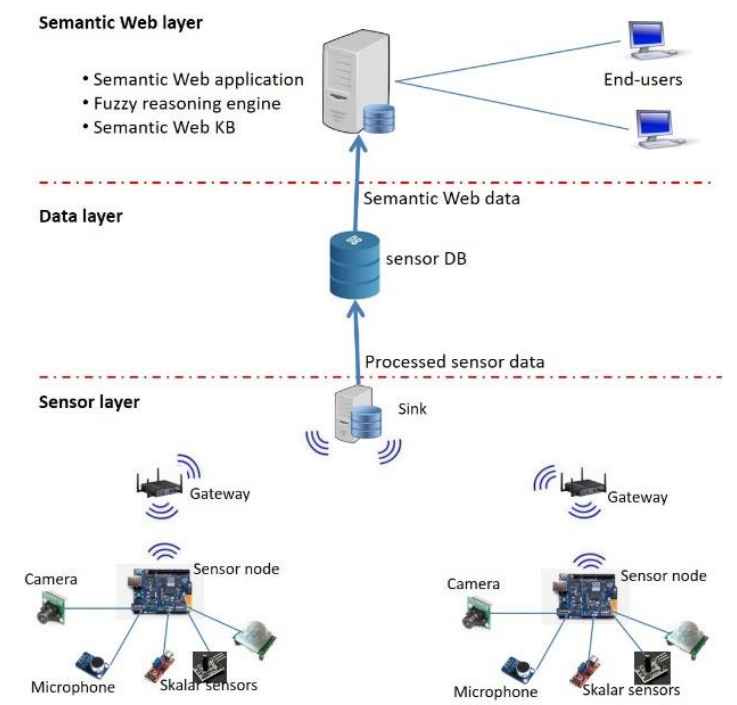

Figure 1: System architecture.

\subsection{Sensor Layer}

The sensor layer is the lowest layer of the architecture. In this layer, by deploying sensors in a selected area, environmental conditions and living things in the environment are monitored to recognize the objects. As shown in Figure 1, this layer includes wireless multimedia sensor nodes with multimedia sensors (camera and microphone) and scalar sensors (passive infrared sensors - PIR, acoustic and vibration). Normally, multimedia sensors are kept in sleep mode to reduce power consumption. On the other hand, the scalar sensors are always in active mode. The cameras and microphones are chosen to be woken up according to the measurements of the scalar sensors. When the camera and microphone are turned on, audio and video are processed using automatic learning methods and the results are fused at the sensor node. Then, the generated information is transferred to the sink via gateways and a more complex fusion is performed here to accurately detect and recognize the objects in the monitored area of the established WMSN. More information about the WMSN is available in [12]. This pre-processed data is then transferred to the data layer in the middle of the architecture.

\subsection{Data Layer}

The pre-processed sensor data extracted from the sensor layer is stored in the data layer to be used in the semantic web layer later. The data stored in this layer includes information about detected entities, such as entity type and timestamp, as well as information about sensors, such as their positions. For example, the data stored in the data layer for the case study described in Section 4 contains latitude, longitude, altitude, sensors in the neighborhood, time stamp, and type of entity detected.

The position of the sensor expressed in latitude, longitude and altitude, as well as the type of entity with time stamp play an important role in the event detection performed by the Semantic Web layer. The type of entity can be, for example, a person, a dog or a car. The data layer server sends a notification to the semantic web layer about the arrival of a new WMSN entry and triggers the operation of the semantic web layer.

\subsection{Semantic Web Layer}

This layer is responsible for managing all operations associated with the Semantic Web. Activity reasoning is performed at this layer. It has three main components: Web application core, WMSN semantic $\mathrm{KB}$ and fuzzy semantic reasoning engine. It converts the data taken from the data layer into semantic tuples (subject, predicate, and object) in accordance with designed ontology. In addition, client requests are processed at this layer.

One of the purposes of using the Semantic Web is to facilitate the integration of this system with other applications. With the idea of the Semantic Web, RDF connects subjects by moving the idea of Web 2.0 to link documents a little further [26]. RDF provides this 
by giving a unique resource identifier (URI) to each subject. Thus, if the same subject is mentioned on different web pages, the data of these two subjects can be related (linked) to each other. In addition, this architecture can be used to make WMSN data available in a well-defined format with a defined ontology for any other system to use this data.

The semantics at semantic web layer is based on a fuzzy abstract ontology illustrated in Figure 2. This ontology is designed not only to cover the data obtained from the sensors, but also to allow the input and output membership functions to be implemented in order to perform fuzzification and record the results as triple compatible with the ontology. The fuzzification of the required data elements is performed before storage.

The fuzzy semantic reasoning engine is responsible for the inference process. Inference is the process of obtaining new semantic tuples from existing ones. It automatically passes over the tuples after each new tuple is inserted into the WMSN knowledge base according to the ontology and attempts to extract a new activity detection.

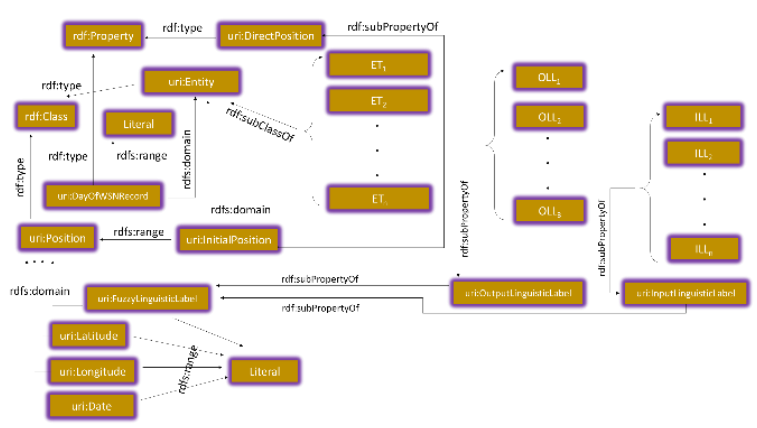

Figure 2: Fuzzy ontology design at semantic layer.

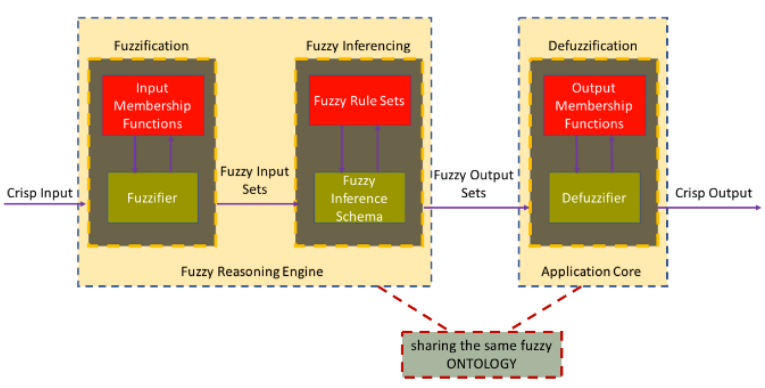

Figure 3: Fuzzy logic controller implementation on semantic web reasoning.

The fuzzy semantic reasoning engine is also responsible for managing fuzzy inferencing process using input membership functions and fuzzy inference rule set according to the ontology, which differs from the classical rule-based reasoning of the Semantic Web, as seen in Figure 3. Figure 4 shows the fuzzy rule set used by the fuzzy inferencing system. In this figure, $I L L_{0,1, . ., n}$ refers to input linguistic values of different input state variables (ISV), while Activity $_{0,1, ., n}$ refers to different values (e.g. walking, running, etc.) that the output state variable can have, in other words, the type of activity to be detected. Furthermore, there is a confidence coefficient $c$ in $[0,1]$ for each rule. The confidence parameter can increase or reduce the effect of that rule by multiplying the output membership degree of the detected activity with the confidence coefficient before defuzzification.

Prior to fuzzy inferencing, crisp inputs are fuzzified using related membership functions. Membership functions usually have trapezoidal, reverse trapezoidal or triangular forms. These equations are implemented within the membership function rules and the fuzzification is completed by using these equations to calculate the ILL $L_{x y}$ (InputStateVar ${ }_{x}$ ), $I L L_{t z}\left(\right.$ InputStateVar $_{t}$ ) input linguistic labels and their membership degrees. For example, Figure 5 shows how a trapezoidal membership function belonging to $I L L_{x y}$ varying according to uri:date is implemented in the fuzzy rule engine.

\begin{tabular}{|c|c|c|c|c|c|c|}
\hline Rule & InputStateVar 0 & InputStateVar 1 & $\ldots$ & InputStateVar $\mathbf{n}$ & Activity & Confidence \\
\hline$R_{0}$ & $I L L_{00}$ & $I L L_{10}$ & $\ldots$ & $I L L_{n 0}$ & Activityo $_{0}$ & $c_{0}$ \\
\hline$R_{1}$ & $I L L_{01}$ & $I L L_{11}$ & & $I L L_{n 1}$ & Activity $_{1}$ & $c_{1}$ \\
\hline . & . & . & $\ldots$ & & . & \\
\hline & & & & & & \\
\hline . & . & . & $\ldots$ & . & . & . \\
\hline$R_{a-2}$ & $I L L_{0_{a-2}}$ & $I L L_{1 a-2}$ & & $I L L_{n a-2}$ & Activity $_{a-2}$ & $c_{a-2}$ \\
\hline$R_{a-1}$ & $I L L_{0_{a-1}}$ & $I L L_{1 a-1}$ & $\ldots$ & $I L L_{n a-1}$ & Activity $_{a-1}$ & $c_{a-1}$ \\
\hline$R_{a}$ & $I L L_{0_{a}}$ & $I L L_{1 a}$ & & $I L L_{n_{a}}$ & Activity $_{a}$ & $c_{a}$ \\
\hline
\end{tabular}

Figure 4: Structure of fuzzy rule base

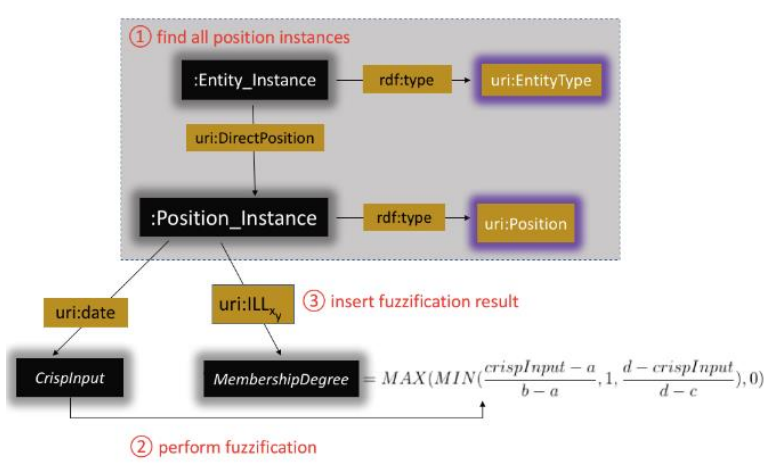

Figure 5: Fuzzification with the ontology.

After fuzzification, the fuzzy inference process begins with the fuzzy rule set given in Figure 4. In this process, activities and related membership degrees are calculated with one or many triggered rules. The reasoning rules must be defined before the initialization phase of the system.

The semantic Web application manages and coordinates the entire Semantic Web Layer process. Communication between the data layer and the semantic Web layer is bidirectional. The initialization 
of the communication depends on a parameter defined in the core of the Web application. In online mode, the instantaneous data of the WMSN is monitored and the sensor database in the data layer informs the semantic web application of the availability of new WMSN data. In offline mode, the core of the Web application establishes the communication and requests the previously stored WMSN data. Communication with end users is also handled by this application. New data from the data layer is converted to semantic WMSN data by the core of the web application. It also activates the fuzzy semantic reasoning engine to determine activities. At the end, the defuzzification process is executed as shown in Figure 3. With the defuzzification process, the system completes the activity detection. Defuzzification is done using the center of gravity method given in Equation (1).

$$
\operatorname{CoG}=\frac{\sum_{a=1}^{N}\left(z_{a}\right) * u_{c}\left(z_{a}\right)}{\sum_{a=1}^{N} u_{c}\left(z_{a}\right)}
$$

\section{Case Study: Border Safety Informer}

National security is one of the most important issues of this century. Soldiers protecting borders can face a sudden attack or other activities. The Border Safety Informer (BSI) is designed to monitor the controlled field and inform troops waiting at the border post by calculating the risk of danger. The activity detection architecture defined in Figure 1 is implemented with the appropriate software components, as shown in Figure 6. In this scenario, we assume that there is a WMSN composed of thousands of sensors around the border station and that the data detected by the multimedia and scalar sensors is pre-processed for the detection of entities at the sensor layer. Then this data is transferred to the upper layers for activity detection.

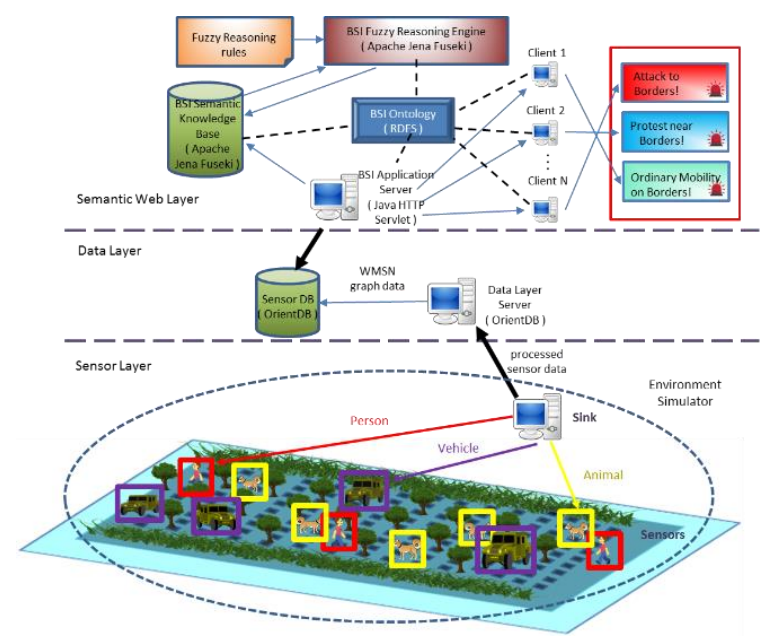

Figure 6: Border Safety Informer System.

The sensor layer is simulated by a program called the Environment Simulator (ES) which deploys a WMSN according to the defined parameters. After parameter definitions, the ES creates a WMSN and inserts related data to the sensor DB. It can produce sensor data according to the given scenarios. Entities such as Person, Animal, Vehicle, Group of People and Vehicle with Group of People can be created. When a simulation is started according to the given scenario, the instantaneous positions of the simulated entities with their time stamps are inserted into the sensor database as if there is a real WMSN.

The data layer consists of a sensor database running on a server. The WMSN data generated by the ES is saved in the Sensor DB via the data layer server. Since the data produced by the simulator is in graph model [20], we have a big graph database system at the data layer. In the case study, OrientDB, as a graph database system, is used as the sensor DB. The position information (latitude, longitude) of each sensor is the first data recorded in the Sensor DB graph store. After launching a simulation, the type and position of the entities with a timestamp and ID of the sensor are inserted in the sensor database. OrientDB has a push notification service. The BSI application server which is in the Semantic Web layer registers to push notification service of OrientDB. A notification is sent to the BSI application server to initiate data transfer when an update is made in the sensor database.

The data retrieved by the BSI application is converted to semantic Web tuples (subject, predicate and object) according to the designed ontology. As soon as a new semantic web tuple is inserted into the semantic KB, the fuzzy reasoning engine is activated and initiates the fuzzification process and the inference process for each data entry. In order to perform fuzzification, the implementations of the membership functions shown in Figure 7 is assigned to the fuzzy reasoning engine via the input rule definition file provided during the initialization phase. The membership functions are applied to the timeOfDay and closeness input state variables. timeOfDay can take two linguistic values (daytime and night) with a certain degree of membership. Closeness can receive three linguistic values (very close, close and far) with a certain membership degree. On the other hand, there are also crisp input state variables. One of them is movingDirection with two linguistic values called gettingCloser and goingFar. The other is detectedEntityType that have five linguistic labels: person, animal, vehicle, grpOfPeople, vehicleWithGrpOfPeople.

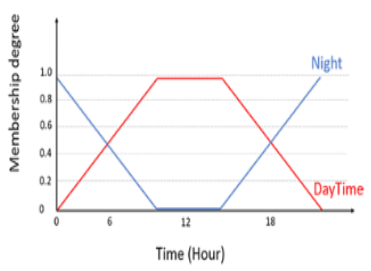

(a) Time of day

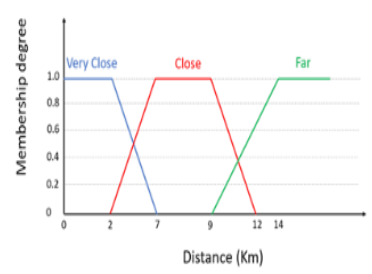

(b) Distance to target
Figure 7: Input membership functions. 
To reason over the Web with semantic techniques, the Apache Fuseki framework is used. Fuseki acts as a SPARQL server where select, insert, update, or delete operations can be performed on semantic web tuples over HTTP [2]. During reasoning, the linguistic value of the dangerRate output state variable and its membership degrees are calculated. DangerRate can take three linguistic values namely ordinaryMobility, terrorAttack or protest. A total of $5 \times 3 \times 2 \times 2=60$ different cases is evaluated for five entityType, three closeness, two timeOfDay and two movementDirection values defined in the fuzzy rule base. In order to perform defuzzification, the method described in [10] and [11] is adapted for the BSI application. After the inferencing process, the output linguistic values of the dangerRate state variable and calculated membership degrees with confidence coefficients are received from the WMSN KB by the BSI application. Then, the application calculates a crisp dangerRate value in percentage as formulated in Equation (1) using the dangerRate output membership function given in Figure 8. Then, BSI shares deduced activity information with clients.

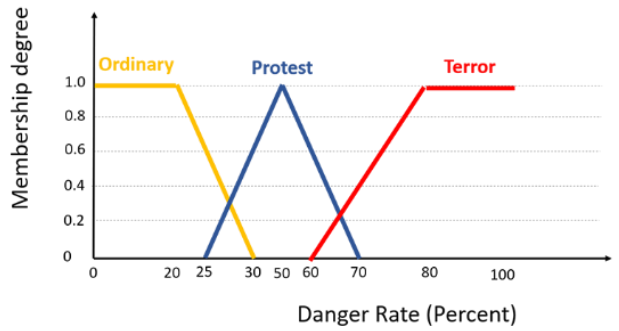

Figure 8: Output membership function.

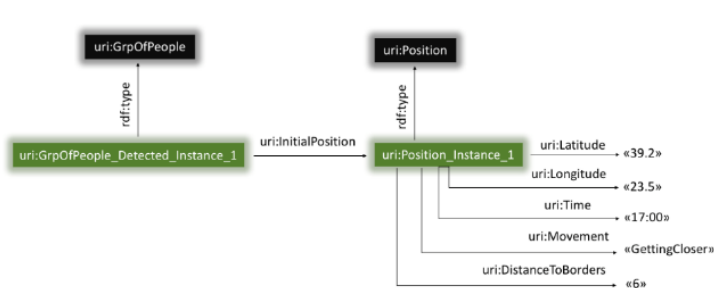

Figure 9: Assertional ontology of semantic WMSN $\mathrm{KB}$.

For example; assume that the BSI application reads sensor data from the data layer and inserts it into the WMSN KB in accordance with the ontology given in Figure 2 . Then, the fuzzy reasoning engine is activated and the fuzzification process begins. Membership degrees are computed as below using membership functions shown in Figure 9. Fuzzy input values are obtained as a result of the fuzzification process after membership calculations as given below and the final version of the ontology on the WMSN KB becomes as in Figure 10.

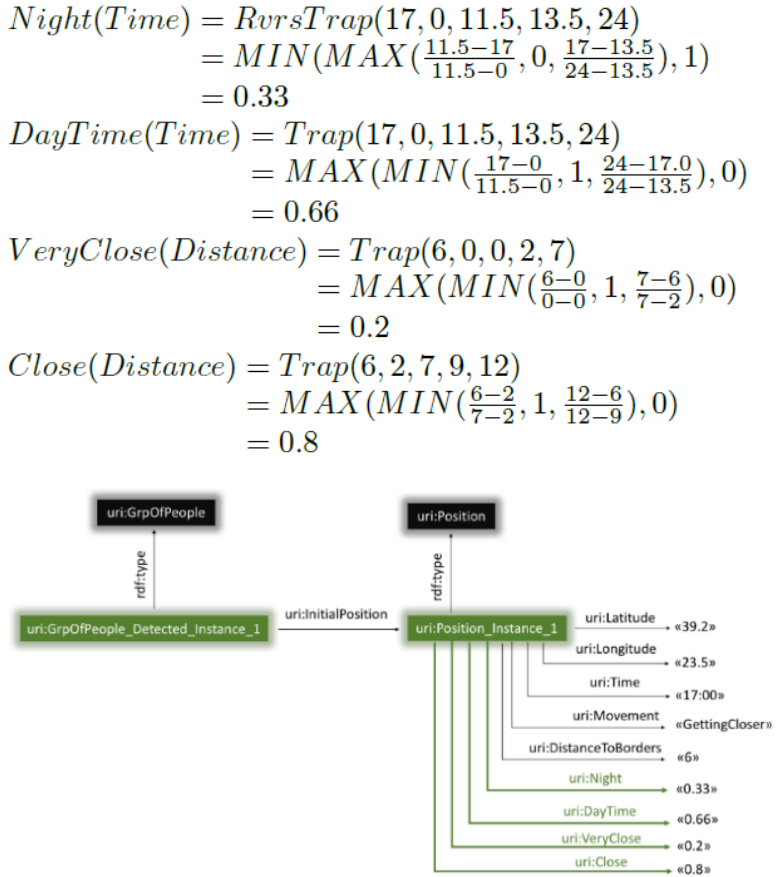

Figure 10: Assertional ontology of semantic WMSN $\mathrm{KB}$ after fuzzification.

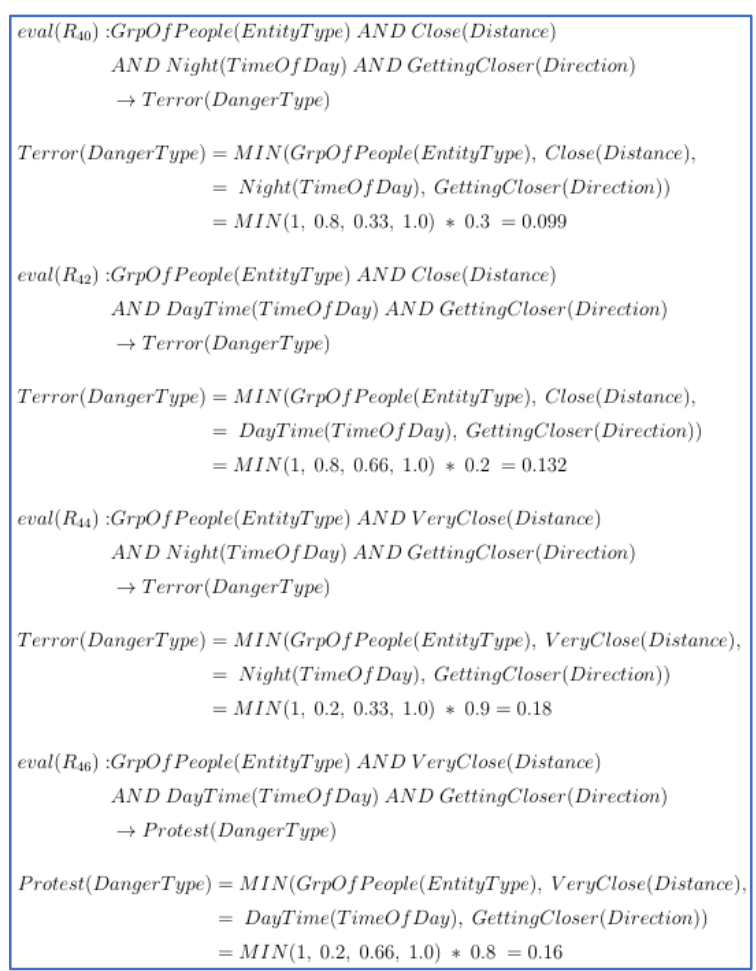

Figure 11: Fuzzy inferencing calculation.

After fuzzification, fuzzy inference starts by the fuzzy reasoning engine and the related rules are fired. As a result of firing of rules, output membership degrees are calculated. The values obtained after this calculation are multiplied by the confidence coefficients of related rules as shown in Figure 11. At 
the end, the output membership degrees are calculated and defuzzification is performed by the BSI application. The final version of the WMSN KB is illustrated in Figure 12. The output membership degrees obtained from four triggered rules are defuzzified and a crisp danger rate is calculated. The calculation of the defuzzification is performed according to Equation (1) as shown in Figure 13.

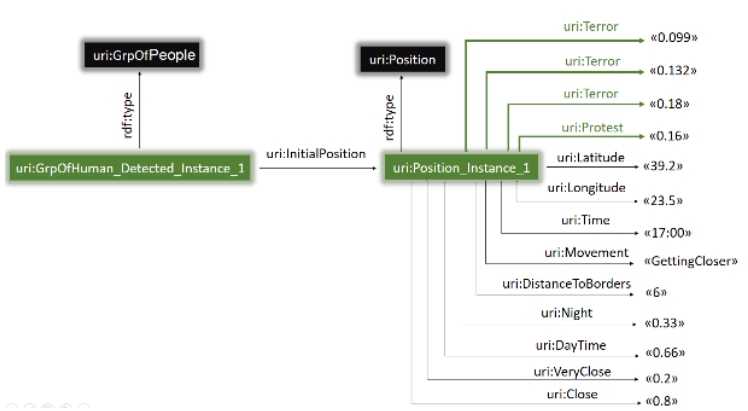

Figure 12: Assertional ontology of semantic WMSN $\mathrm{KB}$ after fuzzy inferencing.

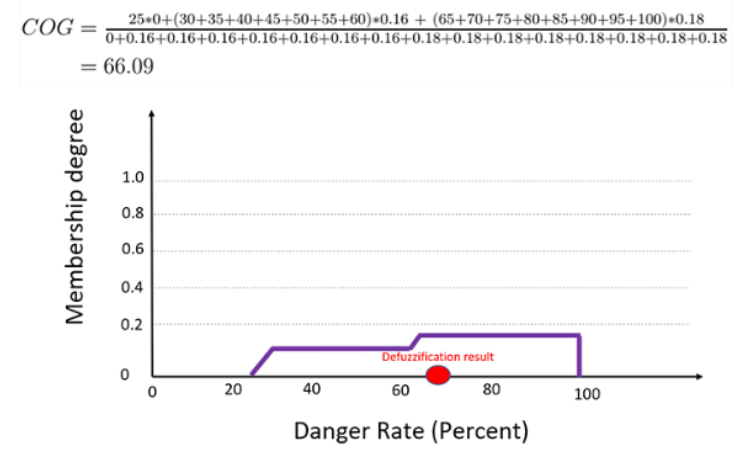

Figure 13: Calculation of defuzzification with CoG.

\section{Conclusion}

The main idea put forward in this study is that activity detection can be done with the Semantic Web. Our study focuses on the use of semantic Web reasoning to detect activities in a WMSN. In addition, fuzzy set theory is used in conjunction with semantic web reasoning. In this way, our approach gives the reasoning mechanism of the semantic Web the possibility of inferring more similar to humans. Through the use of the Semantic Web with a designed ontology, sensor data and fuzzy reasoning are shared with other third-party applications in a well-defined machine-readable format. The proposed architecture is assessed using a case study called Border Safety Informer (BSI). Our implementation provides promising results for the use of the proposed architecture under real-world conditions.

\section{References}

[1] I. F. Akyildiz, T. Melodia, K. R. Chowdhury, A survey on wireless multimedia sensor networks, Computer Networks, 51 (2007) 921-960.

[2] The Apache Software Foundation, Apache Jena. [Online]. Available: https://jena.apache.org/, [Accessed: 07/10/2018].

[3] U. Arora, A. Kumar, Architecture for Raw Data Processing at Sensor Nodes in Semantic Wireless Sensor Networks, International Journal of Computer Applications, 55 (2012) 24-26.

[4] G. R. Berkenbrock, C.M. Hirata, F.G.A. de Oliveira Junior, J.M.P. de Oliveira, Applying Semantic Web Services and Wireless Sensor Networks for System Integration, in: M. Ulieru, P. Palensky, R. Doursat (Eds.), IT Revolutions 2008. LNCS, Social Informatics and Telecommunications Engineering, vol 11. Springer, Berlin, Heidelberg, 2009.

[5] T. Berners-Lee, J. Hendler, and O. Lassila. "The semantic web," Scientific American, vol.284, no.5, pp.34-43, 2001.

[6] K. A. Bispo, S. R. Nelson, and P. R. F. Cunha, "A semantic solution for saving energy in wireless sensor networks," IEEE Symposium on Computers and Communications (ISCC), 2012.

[7] E. Brands, Integration of WSN in Semantic Web. MS Thesis. Universitat Politècnica de Catalunya, Spain (2014).

[8] N. Cho and E. Kim, Enhanced voice activity detection using acoustic event detection and classification, IEEE Transactions on Consumer Electronics, 57 (2011) 196-202.

[9] S-H. Choi, B-K. Kim, J. Park, C-H. Kang, and DS. Eom, An implementation of wireless sensor network, IEEE Transactions on Consumer Electronics, 50 (2004) 236-244.

[10] P. Cingolani, J. Alcala-Fdez, jFuzzyLogic: a robust and flexible Fuzzy-Logic inference system language implementation, in: 2012 IEEE International Conference on Fuzzy Systems, Brisbane, QLD, 2012, pp. 1-8.

[11] P. Cingolani, J. Alcala-Fdez, jFuzzyLogic: a java library to design fuzzy logic controllers according to the standard for fuzzy control programming, International Journal of Computational Intelligence Systems, 6 (2013).sup1, 61-75.

[12] M. Civelek, A. Yazici, Automated Moving Object Classification in Wireless Multimedia Sensor 
Networks, IEEE Sensors Journal, 17 (2017) 11161131.

[13] G. Civitarese, C. Bettini, T. Sztyler, D. Riboni, H. Stuckenschmidt, NECTAR: Knowledge-based collaborative active learning for activity recognition, in: 2018 IEEE International Conference on Pervasive Computing and Communications, Athens, Greece, March 19-23, 2018.

[14] L. Gao, M. Bruenig, J. Hunter, Estimating fire weather indices via semantic reasoning over wireless sensor network data streams, International Journal of Web and Semantic Technology, 5 (2014) 1-20.

[15] J. Gómez-Romero, M. Á. Patricio, J. García, and J. M. Molina, Context-based reasoning using ontologies to adapt visual tracking in surveillance, in: 6th IEEE International Conference on Advanced Video and Signal Based Surveillance (AVSS 2009), 2009, pp. 226-231.

[16] J. Gómez-Romero, M. Á. Serrano, J. García, J. M. Molina, and G. Rogova, Context-based multilevel information fusion for harbor surveillance, Inf. Fusion, 21 (2015), 173-186.

[17] H. M. S. Hossain, N. Roy, M. A. A. H. Khan, Active learning enabled activity recognition, in: 2016 IEEE International Conference on Pervasive Computing and Communications (PerCom), Sydney, NSW, 2016, pp. 1-9.

[18] V. Huang, M. K. Javed, Semantic sensor information description and processing, 2008 Second International Conference on Sensor Technologies and Applications (sensorcomm 2008), Cap Esterel, 2008, pp. 456-461.

[19] K. Kapitanova, S.H. Sang, K-D. Kang, Using fuzzy logic for robust event detection in wireless sensor networks, Ad Hoc Networks, 10 (2012) 709-722.

[20] C. Kucukkececi, A. Yazici, Big Data Model Simulation on a Graph Database for Surveillance in Wireless Multimedia Sensor Networks, Big Data Research, 11 (2018) 33-43.

[21] Y. J. Lee, J. Trevathan, I. Atkinson, W. Read, The Integration, Analysis and Visualization of Sensor Data from Dispersed Wireless Sensor Network Systems Using the SWE Framework, Journal of Telecommunications and Information Technology, 4 (2015) 86-97.

[22] H. Liu, A. Gegov, Rule based systems and networks: Deterministic and fuzzy approaches, in: 2016 IEEE 8th International Conference on Intelligent Systems (IS), Sofia, 2016, pp.316-321.

[23] A. Milenkovic, C. Otto, E. Jovanov, Wireless sensor networks for personal health monitoring: Issues and an implementation, Computer communications, 29 (2006) 2521-2533.

[24] A. Moraru, D. Mladenic, A framework for semantic enrichment of sensor data, Journal of computing and information technology, 20 (2012) $167-173$.

[25] W. Nicolas, Fuzzy classification of online customers, Chapter 2, Springer International Publishing, 2015.

[26] W3C, Resource Description Framework (RDF), [Online]. Available: https://www.w3.org/TR/rdfconcepts/, [Accessed: 07/10/2018].

[27] J. Ye, S. Dasiopoulou, G. Stevenson, G. Meditskos, E. Kontopoulos, I. Kompatsiaris, S. Dobson, Semantic web technologies in pervasive computing: A survey and research roadmap, Pervasive and Mobile Computing, 23 (2015), 125.

[28] L. A. Zadeh, Fuzzy sets, in: G.J.Klir and B.Yuan (Eds.), Fuzzy Sets, Fuzzy Logic, and Fuzzy Systems: Selected Papers by Lotfi A Zadeh, World Scientific, 1996.

[29] A. Zafeiropoulos, D-E. Spanos, S. Arkoulis, N. Konstantinou, N. Mitrou, Data management in sensor networks using semantic web technologies, In: H.Jin, Z.Lv (Eds.), Data Management in Semantic Web, Nova Science Publishers, Inc. 2009. 